\title{
Stay Out of the Blast Radius: Influence of Surgical Masks on Virtual Pedestrian Interactions
}

Marco A. Bühler ( $\nabla$ marco.buhler@mail.mcgill.ca )

McGill University

Sean D. Lynch

McGill University

Trineta Bhojwani

McGill University

Ahlam Zidan

McGill University

Félix Fiset

CIUSSS de la Capitale Nationale

Bradford J. McFadyen

CIUSSS de la Capitale Nationale

Anouk Lamontagne

McGill University

\section{Research Article}

Keywords: blast radius, COVID-19, surgical masks, virtual pedestrian interactions

Posted Date: September 2nd, 2021

DOl: https://doi.org/10.21203/rs.3.rs-852079/v1

License: (c) (1) This work is licensed under a Creative Commons Attribution 4.0 International License.

Read Full License 


\section{Abstract}

To combat the global pandemic caused by COVID-19, a series of mitigation strategies have been proposed by governments around the world. While responses varied across different governing bodies, recommendations such as social distancing and the use of facial masks were nearly universal. Considering that even with restrictions in place, walking in community environments remained an important component of everyday life, these public health recommendations, as well as the anxiety generated by the pandemic, are likely to have influenced pedestrian interactions. In this study, we have examined the effect of facial masks and anxiety related to community ambulation in the context of the COVID-19 pandemic. Using virtual reality, obstacle circumvention strategies in response to approaching pedestrians with and without facial masks were measured in a sample of 11 healthy young individuals. Additionally, a questionnaire was developed and used to gain insights into the participant's behaviours during and after a strict period of restrictions that were in effect before the summer of 2020. Results showed that participants maintained a larger obstacle clearance when virtual pedestrians wore a facial mask. The extent of obstacle clearance was also positively associated with anxiety towards community ambulation in the context of the pandemic. Our findings provide evidence that mask-wearing results in an increase in physical distancing during pedestrian interactions, which may help to reduce the risk of infection. Furthermore, results demonstrate the effects of social context and psychological status on pedestrian interactions and highlight the potential of virtual reality simulations to study locomotion in natural community settings.

\section{Introduction}

In December 2019 the novel coronavirus (i.e., Covid-19) gave rise to a global pandemic ${ }^{1}$. More than a year later, the pandemic continues to grip the world with many countries facing multiple waves of coronavirus infections. Since its inception, various health and safety guidelines have been incorporated by governments around the globe to curb the community spread of the virus. While most guidelines vary across country and state governments, recommendations such as hand washing, social distancing, and wearing a facial mask are widely promoted. As the virus largely spreads by droplet transmission, these recommendations are practical and efficient methods that decrease the likelihood of transmission from infected to non-infected individuals ${ }^{2,3}$.

Despite the restrictions that were implemented throughout the pandemic, walking in community environments remained an essential element of everyday life. During community ambulation, pedestrians often come across obstructions, such as objects or other people, that prompt the execution of walking adjustments maintain a safe clearance distance, and avoid collisions. Proxemics studies indicate that the interpersonal space maintained by pedestrians is influenced by personal, social, and situational factors 4 . For instance, personal factors include impaired sensorimotor functions such as those incurred by older age or stroke. These factors have been shown to result in slower walking speeds, larger safety margins, and increased risk of collision during obstacle circumvention ${ }^{5,6}$. Additional avoidance behaviour 
modulations have been observed during interactions with pedestrians of different sizes ${ }^{7}$ and pedestrians as opposed to a robot ${ }^{8}$. Finally, studies have shown that avoidance strategies differ depending on situational factors such as the direction of obstacle approach ${ }^{9-11}$ and whether one assumes a passive or active role during the interaction ${ }^{12}$.

In the context of the COVID-19 pandemic, it is reasonable to assume that adherence to the health and safety guidelines and the fear of contracting the disease has influenced social interactions ${ }^{13,14}$. Indeed, recent studies showed that barrier gestures, such as wearing a facial mask, influence interpersonal distances. Accordingly, an internet-based experiment examined judgments of the appropriate interpersonal distance from a virtual human-like character to engage in a social interaction. Results showed that smaller distances were deemed appropriate when characters were wearing a mask as opposed to unmasked characters ${ }^{15}$. Nevertheless, when measuring the total distance maintained from an experimenter standing in line outside a local business ${ }^{16}$ or the minimum distance maintained from an experimenter walking along a sidewalk ${ }^{17}$, researchers observed that individuals maintained larger interpersonal distances in conditions where experimenters were wearing facial masks. Interestingly, a subsequent survey showed that masked individuals are not perceived as being more likely to transmit COVID-19 when compared to their unmasked counterparts ${ }^{16}$. Instead, participants reported maintaining larger distances because masked individuals seem to prefer more distance ${ }^{16}$. While these studies suggest that mask-wearing has an impact on interpersonal distances, the exact nature of this effect remains uncertain and the extent to which it impacts obstacle avoidance strategies during pedestrian interactions remains to be elucidated.

A recent review also demonstrated a higher prevalence of adverse psychiatric symptoms, such as fear and anxiety, when compared to pre-pandemic levels ${ }^{18}$. The study further showed that frequent exposure to news related to COVID-19, mandatory quarantine, unexpected unemployment, and general uncertainty regarding the outbreak was associated with adverse psychological outcomes ${ }^{18}$. While a pre-pandemic study suggests that individuals with a higher degree of social anxiety prefer larger interpersonal distances when a virtual human approaches their current position ${ }^{19}$, the extent to which anxiety symptoms caused by the COVID-19 pandemic influence collision avoidance behaviour, leading to larger interpersonal distances while walking, remains unknown.

Several quantitative measures are used to better understand the complex nature of pedestrian interactions in community settings. These measures describe walking trajectory, relative distances, walking speed, and even gaze behaviour ${ }^{7,20-22}$. Extracting these measures under ecological yet safe and controlled conditions is very challenging. To address these limitations, virtual reality (VR) has been used extensively as an experimental tool and recent investigations have shown that it can yield locomotor responses during obstacle avoidance that are similar to those observed in the real world ${ }^{23-25}$. In the present study, which took place after the first wave of the pandemic in Canada (July-September 2020), we used an immersive VR set-up allowing for gaze and body movement recording to examine the avoidance strategies adopted by healthy young individuals during interactions with virtual pedestrians (VRPs) with 
and without a facial mask. Furthermore, we explored the relationship between obstacle avoidance outcomes and self-reported levels of frequency of walking in the community and anxiety associated with the COVID-19 pandemic. We hypothesized that participants would maintain larger clearances, as reflected by earlier onsets of trajectory deviation and/or larger minimum distances when avoiding a VRP wearing vs. no wearing a facial mask. We further hypothesized that larger obstacle clearances would be associated with a lower frequency of walking in the community and/or higher levels of anxiety towards community ambulation during the pandemic. Lastly, we hypothesized that when interacting with an interferer wearing a facial mask, participants would fixate their gaze on the interferer for longer periods and even longer fixations would be maintained on the interferer's head.

\section{Results}

Traces representing participant's walking trajectories from the sample are displayed in Fig. 1. From these graphics, it can be observed that in the presence of a facial mask, mediolateral path deviations seem to start earlier and reach larger magnitudes.

Significant differences due to direction of approach were observed for minimum distance $\left(\chi^{2}(2,405)=\right.$ $8.8, \rho=0.01)$, onset distance $\left(\chi^{2}(2,357)=9.28, \rho=0.009\right)$, and maximum deviation $\left(\chi^{2}(2,357)=7.08, \rho=\right.$ $0.02)$. Post hoc comparisons revealed that trajectory adjustments in the middle obstacle approach condition, when compared to that for the left and right approaches, was characterized by smaller minimum distances (left: $\Delta=0.10 \mathrm{~m}, \sigma \overline{\mathrm{x}}=0.01, \rho<0.0001 \mid$ right: $\Delta=0.06 \mathrm{~m}, \sigma \overline{\mathrm{x}}=0.02, \rho<0.01$ ), larger onset distances (left: $\Delta=0.33 \mathrm{~m}, \sigma \overline{\mathrm{x}}=0.07, \rho<0.0001$ | right: $\Delta=0.41 \mathrm{~m}, \sigma \overline{\mathrm{x}}=0.07, \rho<0.0001$ ), and larger maximum deviations (left: $\Delta=0.10 \mathrm{~m}, \sigma \overline{\mathrm{X}}=0.02, \rho=0.0001$ | right: $\Delta=0.11 \mathrm{~m}, \sigma \overline{\mathrm{X}}=0.03, \rho=0.0004$ ). For all these outcome measures, no statistically significant differences were observed when contrasting the left and right VRP approach conditions $(\rho>0.05)$.

When considering walking speed adaptations, the main effect of direction of approach was observed for minimum walking speed $\left(\chi^{2}(2,405)=8.57, \rho=0.01\right)$ and average walking speed $\left(\chi^{2}(2,405)=7.19, \rho=\right.$ $0.02)$, but not for maximum walking speed $\left(\chi^{2}(2,405)=3.01, \rho=0.22\right)$. Post-hoc comparisons showed that responses to a VRP approaching from the middle were characterized by larger minimum ( $\Delta=$ $0.14 \mathrm{~m} / \mathrm{s}, \sigma \overline{\mathrm{X}}=0.02, \rho<0.0001)$ and average $(\Delta=0.07 \mathrm{~m} / \mathrm{s}, \sigma \overline{\mathrm{X}}=0.01, \rho<0.0001)$ walking speeds when compared to the right condition. When contrasting the left and right obstacle approach conditions, the former resulted in larger minimum $(\Delta=0.09 \mathrm{~m} / \mathrm{s}, \sigma \overline{\mathrm{x}}=0.03, \rho=0.04)$ and average $(\Delta=0.04 \mathrm{~m} / \mathrm{s}, \sigma \overline{\mathrm{x}}=0.01$, $\rho=0.02)$ walking speeds. Lastly, neither minimum $(\rho=0.09)$ and average $(\rho=0.07)$ walking speeds significantly differed between the left and middle obstacle approach conditions.

The analysis of gaze fixation outcomes revealed a statistically significant main effect of direction of approach for the duration of fixation on the approaching $\operatorname{VRP}\left(X^{2}(2,373)=6.07, \rho=0.04\right)$ and on other VRP $s\left(\chi^{2}(2,319)=9.43, \rho=0.009\right)$. Post-hoc analyses showed that fixations on the approaching VRP were longer for the middle vs. left $(\Delta=14.89 \%, \sigma \overline{\mathrm{X}}=3.65, \rho=0.0001)$ and right $(\Delta=11.45 \%, \sigma \overline{\mathrm{X}}=3.26, \rho=$ 0.001) approach conditions, but did not differ between the left vs. right approach conditions $(\rho=0.30)$. In 
contrast, longer fixation durations were on other VRPs were observed when comparing the middle to the left $(\Delta=17.13 \%, \sigma \overline{\mathrm{x}}=1.99, \rho<0.0001)$ and right $(\Delta=14.30 \%, \sigma \overline{\mathrm{x}}=2.96, \rho<0.0001)$ obstacle approach conditions. Fixations on the other VRPs also did not differ when contrasting the left and right obstacle approach conditions $(\rho=0.39)$. For fixations on the head of the approaching VRP, no significant differences due to the direction of obstacle approach were observed $\left(\chi^{2}(2,219)=2.60, \rho=0.27\right)$.

With respect to the VR simulation, participants reported experiencing strong feelings of spatial presence inside the virtual environment $(\bar{x}=8 / 10, \sigma=0.89)$. As for the questionnaire regarding the frequency of community ambulation during the pandemic and associated anxiety, results are presented in Table 1. Participants reported low frequencies of community ambulation during the confinement period $(\bar{x}=2.27 / 9$, $\sigma=0.56)$ and a slight increase on the post confinement period $(\bar{x}=3.64 / 9, \sigma=0.71)$. Additionally, correlations between frequency of community ambulation and measures of obstacle clearance, such as minimum distance (No Mask: $r=0.02, \rho=0.94$ | Mask: $r=-0.19, \rho=0.56$ ) and onset distance (No mask: $r=$ $0.17, \rho=0.61$ | Mask: $r=0.10, \rho=0.76)$, did not reach statistical significance. Regarding anxiety towards community ambulation, the total anxiety score had an average of $15.18 \pm 6.93$ out of a possible maximum score of 30. Participants reported high levels of adherence to the government's confinement measures, none reported contracting COVID-19 or having contact with someone who had been diagnosed with COVID-19. When questioned about the feeling of anxiety when interacting with the VRP in contrast to a real person, most reported feeling less anxious about a third reported no change, and only one reported feeling more anxious. 


\section{Frequency of walking in the community}

Questionnaire items

Mean \pm 1SD

1. During the confinement, how often did you walk outside in an environment that $\quad 1.36 \pm 1.07$ included other people?

2. During the confinement, how often did you go to a store?

$0.63 \pm 0.48$

3. During the confinement, how often did you go to your workplace?

$0.27 \pm 0.86$

4. During the confinement, how often did you follow the news about Covid-19?

$2.18 \pm 0.87$

5. Since the end of the confinement, how often did you walk outside in an

$2 \pm 1.04$ environment that included other people?

6. Since the end of the confinement, how often did you go to a store?

$1 \pm 0.43$

7. Since the end of the confinement, how often did you go to your workplace?

$0.64 \pm 0.98$

8. Since the end of the confinement, how often did you follow the news about Covid19 ?

\section{Anxiety associated with Community walking}

\section{Questionnaire items}

Mean \pm 1SD

9. How anxious are you to take an outdoor walk in a place frequented by other people?

10. How anxious are you to go to a store?

$5.55 \pm 2.46$

11. How anxious are you to go to work?

$4.27 \pm 2.89$

Equal

Less More

12. In relation to outdoor walking, are you more, less anxious than you were 4 during the confinement, or this has not changed?

13. In relation to going to a store, are you more, less anxious than you were during the confinement, or this has not changed?

14. In relation to going to work, are you more, less anxious than you were during the confinement, or this has not changed?

Additional questions

Questionnaire items

Equal Less More

15. Today, how anxious were you to interact with the virtual human compared to a real person?

16. On a scale of 1 to 10 , to what extent do you believe you are complying with the government's confinement measures? 1 implies not complying with measures at all and 10 implies completely adhering to measures. 


\section{Frequency of walking in the community}

18. Have you contracted Covid-19 in the past months?

Yes No

0

11

20. Have you been in contact with someone you know who has been diagnosed with Covid-19?

\begin{tabular}{ll} 
Yes & No \\
\hline 0 & 11
\end{tabular}

22. When passing another individual in the street, do you allow more space between you than before?

\begin{tabular}{cc} 
Yes & No \\
\hline 10 & 1
\end{tabular}

Lastly, strong positive correlations between the sum of anxiety scores and measures of obstacle clearance were observed. Indeed, as displayed in Fig. 3, the total anxiety score was positively correlated with minimum distance for the mask condition $(r=0.75, n=11, \rho<0.01)$, but the correlation was not significant for the no mask condition $(r=0.59, n=11, \rho=0.07)$. For the onset distance, strong positive correlations with the total anxiety score were observed for both the mask $(r=0.86, n=11, \rho<0.01)$ and no mask $(r=0.84, n=11, \rho<0.01)$ conditions.

\section{Discussion}

In this study, and while using a VR paradigm, we showed that facial masks affect pedestrian circumvention when walking in a community environment. We further showed that obstacle clearance outcomes were associated with the level of anxiety but not the frequency of community ambulation in the context of the COVID-19 pandemic. Possible explanations and implications of these findings are presented below.

From all metrics describing obstacle circumvention, onset distance was the only outcome that changed significantly when the VRPs were wearing a facial mask. Indeed, when participants avoided an approaching VRP that was not wearing a facial mask, trajectory deviations started on average at $2.64 \mathrm{~m}$ from the VRP. This anteroposterior distance reflects the point at which pedestrians begin to adapt their walking trajectory in response to an obstruction and its magnitude indicates a critical point at which walking adaptations are deemed necessary to smoothly and safely avoid collisions ${ }^{21}$. In the current experiment, when the approaching VRP wore a facial mask, this onset distance was increased by approximately $0.18 \mathrm{~m}$. This change in onset distance is, in our perspective, meaningful, as unlike other obstacle clearance metrics such as minimum distance and maximum deviation, onset distance is a fairly stable measure that was shown to remain constant despite manipulations of characteristics of the interferer, such as gender, size, body orientation, and pattern of limb movements 7,26 .

Modulations in this onset distance have been reported, however, according to the direction of obstacle approach (Buhler \& Lamontagne, 2018; Souza Silva et al., 2018). In the present study, as well as in previous investigations, onset distance was found to be enlarged in response to head-on approaching pedestrians. This increase in the anteroposterior clearance may reflect the execution of a safer strategy 
that is used in conditions involving a greater risk of collision. Likewise, in the context of this study, which was conducted during the pandemic, it is also possible that observing a VRP wearing a facial mask would make participants more aware of an infection risk. Consequentially, a larger onset distance would allow participants to move away, at a greater distance, from the direction at which the VRP would be exhaling. At this distance, they would be less likely to be in contact with respiratory droplets that could lead to a COVID-19 infection. Considering the results of a recent survey where masked individuals were perceived to prefer larger interpersonal distances ${ }^{16}$, it is also possible that participants may have perceived the VRPs wearing a mask in the same fashion, resulting in an increased onset distance.

Results also show that other measures of obstacle clearance (i.e., minimum distance and maximum deviation) did not differ between the mask vs. no mask conditions. There was, however, a trend towards larger clearances in the mask condition which can be clearly observed in the bar graphs presented in Fig. 4. This observation, combined with the onset distance results discussed above, further highlights how wearing a facial mask in the context of the COVID-19 pandemic influences interpersonal distances. Interestingly, while we expected that longer fixations on the approaching interferer would be maintained in the mask condition, especially on the interferer's head, such an effect was not observed. It is possible that a short fixation on the interferer's head provided sufficient information to bring about changes in obstacle circumvention strategies. The fact that trials where the interferer was wearing a mask were performed in sequence and that other pedestrians in the environment were also wearing a mask may have also minimized the need to visually scan for the presence of a facial mask. Importantly, the fact that a maskinduced effect could be detected in this study adds further support to the use of VR as a valid tool to study movement behavior in the context of social interactions. In previous studies from our laboratory, and even though VR can introduce a distance perception bias (Renner et al., 2013), we have shown that obstacle avoidance behavior is essentially the same in response to virtual vs. real pedestrians, with similar onset distance values and slightly larger minimum distances in VR (Buhler \& Lamontagne, 2018, 2019). It is thus reasonable to assume that the mask-induced changes observed in the present study do reflect a natural behaviour. It cannot be fully excluded, however, that the effects due to the facial mask might be even larger in the physical world where the contamination risk is 'real', especially since $63 \%$ of participants reported feeling less anxious when interacting with the VRPs compared to real pedestrians.

The healthy and young participants tested as part of this study reported a low frequency of community walking during and after the confinement period. Considering the confinement measures that were put in place and the high adherence to these measures that our participants reported, this finding was not unexpected. Interestingly, after the confinement period, the frequency of community ambulation increased but only by a small amount. In agreement with a recent study that compiled mobile device data from 1.62 million anonymous users in 10 metropolitan areas in the United States ${ }^{27}$, results from our questionnaire suggest that while community ambulation increased with the easing of lockdown restrictions, people still show signs of reluctance to leave their homes.

Overall, participants were somewhat anxious towards community ambulation. In parallel, they reported maintaining larger interpersonal distances when interacting with another pedestrian after the pandemic 
started. These observations show that the pandemic and confinement measures influenced self-reported psychological and behavioral metrics related to community ambulation. In addition, we observed strong positive correlations between anxiety and clearance measures during actual obstacle avoidance while walking. These findings corroborate a previous study that showed associations between anxiety and a preference for larger interpersonal distances ${ }^{19}$. Furthermore, the stronger and more consistent (i.e., present for the mask and no-mask conditions) correlations observed between anxiety and onset distance (vs. minimum distance) supports the idea that modulations of the anteroposterior interpersonal distance are implemented as an infection prevention strategy. Taken together, results show that in the context of the pandemic, anxiety towards community ambulation is associated with larger clearances during pedestrian circumvention, which is reflected primarily by an enlargement of anteroposterior interpersonal distances. Furthermore, our findings provide additional evidence that factors beyond sensorimotor processes, such as social context and psychological status, influence complex behaviors such as pedestrian interactions.

All locomotor measures of obstacle circumvention, excluding maximum walking speed and fixation on the head of the approaching VRP, differed significantly according to the direction from which the VRP approached. These variations in circumvention strategies for most have been previously observed $9,24,25,28$ and appear to reflect adaptations according to the bearing angle ${ }^{10}$ or whether the pedestrian passes in front of behind the VRP ${ }^{12}$.

In the context of this experiment, the right and left obstacle approach conditions represent the same bearing angle of $30^{\circ}$ and the middle condition represents a bearing angle of $0^{\circ}$. With respect to the role taken by the participant during the task, while diagonal approaches represent an unresolved situation where they can decide to pass in front or to give-way, the middle condition requires the participant to give way (Olivier et al., 2013). Accordingly, all measures of obstacle clearance (i.e., minimum distance, maximum deviation, and onset distance) showed significant differences between the diagonal obstacle approaches (left or right) and the middle approach, while no differences were observed between the diagonal obstacle approaches. In the middle condition, the observation of larger deviations that started at greater distances from the VRP, yet still resulting in smaller minimum clearances, suggests a higher degree of challenge when compared to the diagonal conditions.

When considering gaze fixation outcomes, differences between the direction of interferer approach were also observed. As depicted in Fig. 4, in the middle condition, participants maintained longer fixations on the approaching VRP and shorter fixations on the other VRPs. Interestingly, the opposite pattern was observed in the diagonal approach conditions. These findings suggest that rather than fixating their gaze on the approaching interferer, participants were likely looking straight ahead, that is towards their heading direction and/or target. Lastly, we observed larger minimum and average walking velocity in response to the VRPs approaching from the middle and from the left as opposed to the right. While these findings contrast with an earlier observation from our own laboratory where no differences in average walking speed across directions of obstacle approach were observed ${ }^{24}$, it should be noted that differences observed here were very small $(<0.1 \mathrm{~m} / \mathrm{s})$. Nevertheless, it is possible that this difference is a product of a 
choice to give way to diagonally approaching interferers, which involve slower walking speeds. In our previous study, participants chose a front pass strategy in approximately $80 \%$ of the trials involving diagonal approaching interferers. In the present study, only $13.7 \%$ of the diagonal approaching interferers were circumvented with a front pass strategy. While we did observe differences, their magnitude is small and in alignment with prior observations which demonstrated that crossing pedestrians at obtuse angles (e.g., ahead of the pedestrian) requires mainly adjustments of trajectory as opposed to walking speed ${ }^{10}$.

We investigated the obstacle circumvention strategies of healthy young participants in response to VRPs with and without facial masks and approaching from different directions. We observed that participants increased their anteroposterior clearance (i.e., onset distance) when VRPs wore a facial mask which allowed them to move away from the VRP's walking path at a greater distance. This larger clearance could be a product of social factors related to a heightened awareness of an infection risk due to exposure to respiratory droplets, to an assumption that the approaching pedestrians prefer larger interpersonal distances or both. Lastly, participants reported feelings of anxiety towards community ambulation, and greater anxiety was associated with larger obstacle clearances, which suggests that those who feel more anxious towards community ambulation during the pandemic confinement period use this obstacle clearance strategy to reduce the risk of a COVID-19 infection. These results provide a better understanding of how the context surrounding the COVID-19 pandemic affects locomotor circumvention strategies, possibly due to an increase in anxiety related to community ambulation. Results further demonstrate the importance of considering social and psychological factors when examining complex locomotor behaviors, as well as the potential of VR for studying the influence of these factors on movement behavior in general.

\section{Methods}

\section{Participants}

Eleven healthy young individuals (sex $=6$ female, age $=25.45 \pm 2.54$ years, mass $=65.05 \pm 10.79 \mathrm{~kg}$, height $=167.73 \pm 7.30 \mathrm{~cm}$ ) took part in this study. All participants were right-handed as per the Edinburgh Handedness Inventory ${ }^{29}$, had normal or corrected-to-normal visual acuity according to the EDTRS eye chart (equal or above to LogMAR of $0^{30}$ ), and intact cognitive function (score $\geq 26$ out of 30 on the Montreal Cognitive Assessment). Exclusion criteria included conditions that could interfere with locomotion, as well as a positive test for COVID-19, contact with someone who tested positive for COVID19 , or traveling outside the country within two weeks prior to data collection. Written informed consent was obtained from all participants prior to data collection and the study was approved by the Research Ethics Board of the Centre for Interdisciplinary Research in Rehabilitation of Greater Montreal (CRIR). All methods were performed in accordance with the relevant guidelines and regulations, including the Declaration of Helsinki.

\section{Experimental set-up and procedure}


This study employed a block repeated-measure experimental design. The data for each participant were collected in one session at the Virtual Reality and Mobility Laboratory of the Jewish Rehabilitation Hospital-CISSS-Laval between the 10th of July and 25th of September 2020, after the first wave of the COVID-19 pandemic in Canada.

Participants were assessed while walking in a virtual environment representing a local subway station and avoiding a collision with a VRP approaching from different directions. More specifically, a theoretical point of collision (TPC), invisible to the participants, was set at $3.5 \mathrm{~m}$ from the participant's initial position. The TPC represented the location at which a collision would occur if the participant failed to perform the necessary walking adjustments to avoid a collision. Three female VRPs, acting as interferers, stood in an arc fashion at $0^{\circ}$ (straight ahead) as well as $30^{\circ}$ to the left and right of the TPC, with a radius of $3 \mathrm{~m}$ (Fig. 4). The experiment comprised of 3 different trial types (experimental, catch and control trials). In experimental trials, as the participant reached $0.5 \mathrm{~m}$ of forward walking, one of the three VRPs started to walk towards the TPC while the others took one step forward and then walked away, creating three possible directions of VRP approach (left, middle, or right). In catch trials, all VRPs took one step forward and walked away while in control trials, the environment was devoid of VRPs. The purpose of catch trials was to reduce task predictability while that of control trials was to provide a measurement of walking speed and walking trajectory in the absence of interferers. During each trial, text messages informed participants of the time to start ("Go") and stop walking ("Stop"), as well as any collision with the approaching VRP ("Collision"). Participants were instructed to walk straight ahead towards the subway map, presented in the far space, and to avoid a collision with an approaching VRP, if any.

The VRPs and environment were created in Autodesk Maya ${ }^{\mathrm{TM}}$ and controlled using the Unreal engine ${ }^{\circledR}$ 4.24. They were visualized through an immersive head-mounted display (HMD) with an integrated eyetracking system (HTC Vive Pro Eye). Participants' head and eye positions were recorded from the HMD at $90 \mathrm{~Hz}$ and used to compute obstacle circumvention measures. The VRPs were animated using motioncaptured data of three different actors and walked at an approximate speed of $1.39 \mathrm{~m} / \mathrm{s}$, which is a typical average walking speed for healthy young adults ${ }^{31}$ and was shown to systematically induce a collision avoidance behaviour in a previous study on healthy young participants ${ }^{24}$. Additionally, and for the purpose of future analyses of gait and intersegmental body coordination, full-body kinematics were collected at $120 \mathrm{~Hz}$ using a 12-camera Vicon-512 motion capture system and 40 reflective markers placed on body landmarks of the participants as per the Plug-In-gait model from Vicon.

\section{Data analysis}

\section{Obstacle circumvention measures}

The following outcomes were selected to describe clearance adaptations. Minimum distance was defined as the smallest distance maintained between the participant's position and the VRP's sternum. To obtain the onset distance a linear regression line was fitted to the data from the $0.5 \mathrm{~m}$ mark until the first point, backtracking from the point of minimum distance at which the mediolateral displacement was smaller 
than $25 \%$ of the maximum within the same period. A deviation larger than the $99 \%$ confidence interval of this linear prediction determined the occurrence of a trajectory deviation. In trials with a trajectory deviation, the first frame preceding this deviation at which the first derivative of the lateral component of the participant's position was smaller than zero was obtained, and onset distance was defined as the Euclidean distance between the participant and VRP. Additionally, in trials in which an onset distance was obtained, maximum deviation was calculated as the maximum mediolateral displacement preceding the point of interferer crossing.

To characterize the participant's speed adaptations, we obtained the first point after the participant's initial acceleration. This point was defined as the first segment after the first step at which forward acceleration was equal or lower than zero. Subsequently, from this point to the point of VRP crossing, the minimum, average, and maximum walking speeds were extracted. Lastly, outcomes characterizing gaze behaviour were calculated as the percentage of the time, between $0.5 \mathrm{~m}$ of forward displacement and the point of VRP crossing, during which participants were fixating their gaze on body segments of the approaching VRP's body and the other VRPs. Also, we measured the duration of gaze fixations on the head of the approaching VRP.

\section{Questionnaires}

Questionnaires were administered after the locomotor assessment. A single-item presence questionnaire was employed to measure participants' feeling of presence in VR ${ }^{32}$. Answers to this questionnaire are rated on a $0-10$ scale, with higher values indicating a stronger feeling of presence in VR. Participants also responded to a custom-made, 19-item questionnaire on community walking behavior and related anxiety during the covid-19 pandemic (Table 1). The first 8 questions explored participant's activities during and after the confinement period and had the following response options: $6-7$ days a week (3 points); 3-5 days a week (2 points); 1-3 days a week (1 point); never (0 points). Questions 9 to 14 evaluated the person's level of anxiety associated with walking in community environments. The remaining questions (15-19) provided information on the level of anxiety when interacting with virtual vs. real humans, the extent to which participants complied with the government's confinement measures, whether they had previous exposure to Covid-19 and known contact(s) with someone who has been diagnosed with COVID-19. The last question asked if, after the start of the pandemic, they adopt larger interpersonal distances when passing another person on the street. Scores from different sections were added together to create a score for frequency of community walking during (questions 1 to 3 ) and after (questions 5 to 6 ) the confinement periods, as well as a total anxiety score (questions 9,11 , and 10).

\section{Statistical analysis}

To examine differences in the outcome measures describing participant's obstacle circumvention strategies between the conditions of interest, a generalized estimating equation (GEE) model was used. The model was built using SAS 9.4 and contained two within-subject factors: barrier gesture (mask and no mask), and direction of obstacle approach (left, middle, and right). An exchangeable correlation structure was used to account for within-subject correlations and the significance level was set to $\rho<$ 
0.05. In the case of multiple comparisons, Bonferroni corrections were applied. A Pearson correlation was used to determine the relationship between the sum of anxiety scores from the custom-made questionnaire related to COVID-19 and, the minimum and onset distance.

\section{Declarations}

\section{Acknowledgments}

We would like to thank Dr. Samir Sangani for developing the virtual environment and the virtual avatars, as well as Christian Beaudoin for developing the Unreal program that managed the experiment. Furthermore, we would like to acknowledge that our custom-made questionnaire was a multicentre creation between McGill University and the University of Laval, and we thank Dr. Anne-Hélène Olivier and Dr. Michael Cinelli for their feedback.

This work was supported by the Natural Sciences and Engineering Research Council (NSERC) under Grant RGPIN/04471-2016 and Marco A. Bühler is supported by a doctoral award from the Fonds de recherche du Québec (FRQS).

\section{Author Contributions}

M.A.B., S.D.L., T.B., A.Z., and A.L. conceived the experiment. M.A.B., S.D.L., T.B., and A.Z. conducted the experiment and were involved in the initial drafts of the manuscript. M.A.B processed the data, performed the statistical analysis, and interpreted the results. All authors reviewed the manuscript and gave feedback on its contents. A.L. supervised the study.

\section{Additional Information}

The authors declare no competing interests.

\section{References}

1. Anand, K. B., Karade, S., Sen, S. \& Gupta, R. M. SARS-CoV-2: Camazotz's Curse. Med J Armed Forces India, 76, 136-141 https://doi.org/10.1016/j.mjafi.2020.04.008 (2020).

2. Chu, D. K. et al. Physical distancing, face masks, and eye protection to prevent person-to-person transmission of SARS-CoV-2 and COVID-19: a systematic review and meta-analysis., 395, 19731987 https://doi.org/10.1016/S0140-6736(20)31142-9 (2020).

3. Eikenberry, S. E. et al. To mask or not to mask: Modeling the potential for face mask use by the general public to curtail the COVID-19 pandemic. Infect Dis Model, 5, 293-308 
https://doi.org/10.1016/j.idm.2020.04.001 (2020).

4. Hayduk, L. A. Personal space: Where we now stand. Psychol. Bull, 94, 293-335 https://doi.org/10.1037/0033-2909.94.2.293 (1983).

5. Darekar, A., Lamontagne, A. \& Fung, J. Locomotor circumvention strategies are altered by stroke: I. Obstacle clearance. J Neuroeng Rehabil, 14, 56 https://doi.org/10.1186/s12984-017-0264-8 (2017).

6. Gerin-Lajoie, M., Richards, C. L. \& McFadyen, B. J. The circumvention of obstacles during walking in different environmental contexts: a comparison between older and younger adults. Gait Posture, 24, 364-369 https://doi.org/10.1016/j.gaitpost.2005.11.001 (2006).

7. Bourgaize, S. M., McFadyen, B. J. \& Cinelli, M. E. Collision avoidance behaviours when circumventing people of different sizes in various positions and locations. J Mot Behav, 53, 166-175 https://doi.org/10.1080/00222895.2020.1742083 (2021).

8. Vassallo, C. et al. How do walkers behave when crossing the way of a mobile robot that replicates human interaction rules? Gait Posture, 60, 188-193 https://doi.org/10.1016/j.gaitpost.2017.12.002 (2018).

9. Souza Silva, W., Aravind, G., Sangani, S. \& Lamontagne, A. Healthy young adults implement distinctive avoidance strategies while walking and circumventing virtual human vs. non-human obstacles in a virtual environment. Gait Posture, 61, 294-300 https://doi.org/10.1016/j.gaitpost.2018.01.028 (2018).

10. Huber, M. et al. Adjustments of speed and path when avoiding collisions with another pedestrian. PLoS One, 9, e89589 https://doi.org/10.1371/journal.pone.0089589 (2014).

11. Basili, P. et al. Strategies of locomotor collision avoidance. Gait Posture, 37, 385-390 https://doi.org/10.1016/j.gaitpost.2012.08.003 (2013).

12. Olivier, A. H., Marin, A., Cretual, A., Berthoz, A. \& Pettre, J. Collision avoidance between two walkers: role-dependent strategies. Gait Posture, 38, 751-756 https://doi.org/10.1016/j.gaitpost.2013.03.017 (2013).

13. Harper, C. A., Satchell, L. P., Fido, D. \& Latzman, R. D. Functional Fear Predicts Public Health Compliance in the COVID-19 Pandemic. Int J Ment Health Addict, 1-14 https://doi.org/10.1007/s11469-020-00281-5 (2020).

14. Greenslade, G. erstanding pedestrian decision-making during the COVID-19 pandemic, UWSpace(2021).

15. Cartaud, A., Quesque, F. \& Coello, Y. Wearing a face mask against Covid-19 results in a reduction of social distancing. PLoS One, 15, e0243023 https://doi.org/10.1371/journal.pone.0243023 (2020).

16. Seres, G. et al. Face Masks Increase Compliance with Physical Distancing Recommendations During the COVID-19 Pandemic(2020).

17. Marchiori, M. COVID-19 and the Social Distancing Paradox: dangers and solutions.arXiv: Populations and Evolution(2020). 
18. Xiong, J. et al. Impact of COVID-19 pandemic on mental health in the general population: A systematic review. J Affect Disord, 277, 55-64 https://doi.org/10.1016/j.jad.2020.08.001 (2020).

19. Perry, A., Rubinsten, O., Peled, L. \& Shamay-Tsoory, S. G. Don't stand so close to me: a behavioral and ERP study of preferred interpersonal distance., 83, 761-769 https://doi.org/10.1016/j.neuroimage.2013.07.042 (2013).

20. Huber, M. et al. Adjustments of speed and path when avoiding collisions with another pedestrian.PLOS ONE9 (2014).

21. Gerin-Lajoie, M., Richards, C. L. \& McFadyen, B. J. The negotiation of stationary and moving obstructions during walking: anticipatory locomotor adaptations and preservation of personal space. Motor control, 9, 242-269 (2005).

22. Boulanger, M. \& Lamontagne, A. in 2017 International Conference on Virtual Rehabilitation (ICVR). 12.

23. Olivier, A. H., Bruneau, J., Cirio, G. \& Pettré, J. A. Virtual Reality Platform to Study Crowd Behaviors. Transportation Research Procedia, 2, 114-122 https://doi.org/10.1016/j.trpro.2014.09.015 (2014).

24. Buhler, M. A. \& Lamontagne, A. Circumvention of Pedestrians While Walking in Virtual and Physical Environments. IEEE transactions on neural systems and rehabilitation engineering: a publication of the IEEE Engineering in Medicine and Biology Society, 26, 1813-1822 https://doi.org/10.1109/TNSRE.2018.2865907 (2018).

25. Buhler, M. A. \& Lamontagne, A. Locomotor circumvention strategies in response to static pedestrians in a virtual and physical environment. Gait Posture, 68, 201-206 https://doi.org/10.1016/j.gaitpost.2018.10.004 (2019).

26. Fiset, F., Lamontagne, A. \& McFadyen, B. J. Limb movements of another pedestrian affect crossing distance but not path planning during virtual over ground circumvention. Neurosci. Lett, 736, 135278 https://doi.org/10.1016/j.neulet.2020.135278 (2020).

27. Hunter, R. F. et al. Effect of COVID-19 response policies on walking behavior in US cities. Nature Communications, 12, 3652 https://doi.org/10.1038/s41467-021-23937-9 (2021).

28. Aravind, G. \& Lamontagne, A. Dual tasking negatively impacts obstacle avoidance abilities in poststroke individuals with visuospatial neglect: Task complexity matters!. Restorative neurology and neuroscience, 35, 423-436 https://doi.org/10.3233/RNN-160709 (2017).

29. Oldfield, R. C. The assessment and analysis of handedness: the Edinburgh inventory., 9, 97-113 (1971).

30. Kaiser, P. K. Prospective evaluation of visual acuity assessment: a comparison of snellen versus ETDRS charts in clinical practice (An AOS Thesis). Trans Am Ophthalmol Soc 107, 311-324(2009).

31. Bohannon, R. W. Comfortable and maximum walking speed of adults aged $20-79$ years: reference values and determinants. Age and ageing, 26, 15-19 https://doi.org/10.1093/ageing/26.1.15 (1997).

32. Bouchard, S. et al. in The 3rd IEEE International Workshop on Haptic, Audio and Visual Environments and Their Applications. 59-61. 
Figures

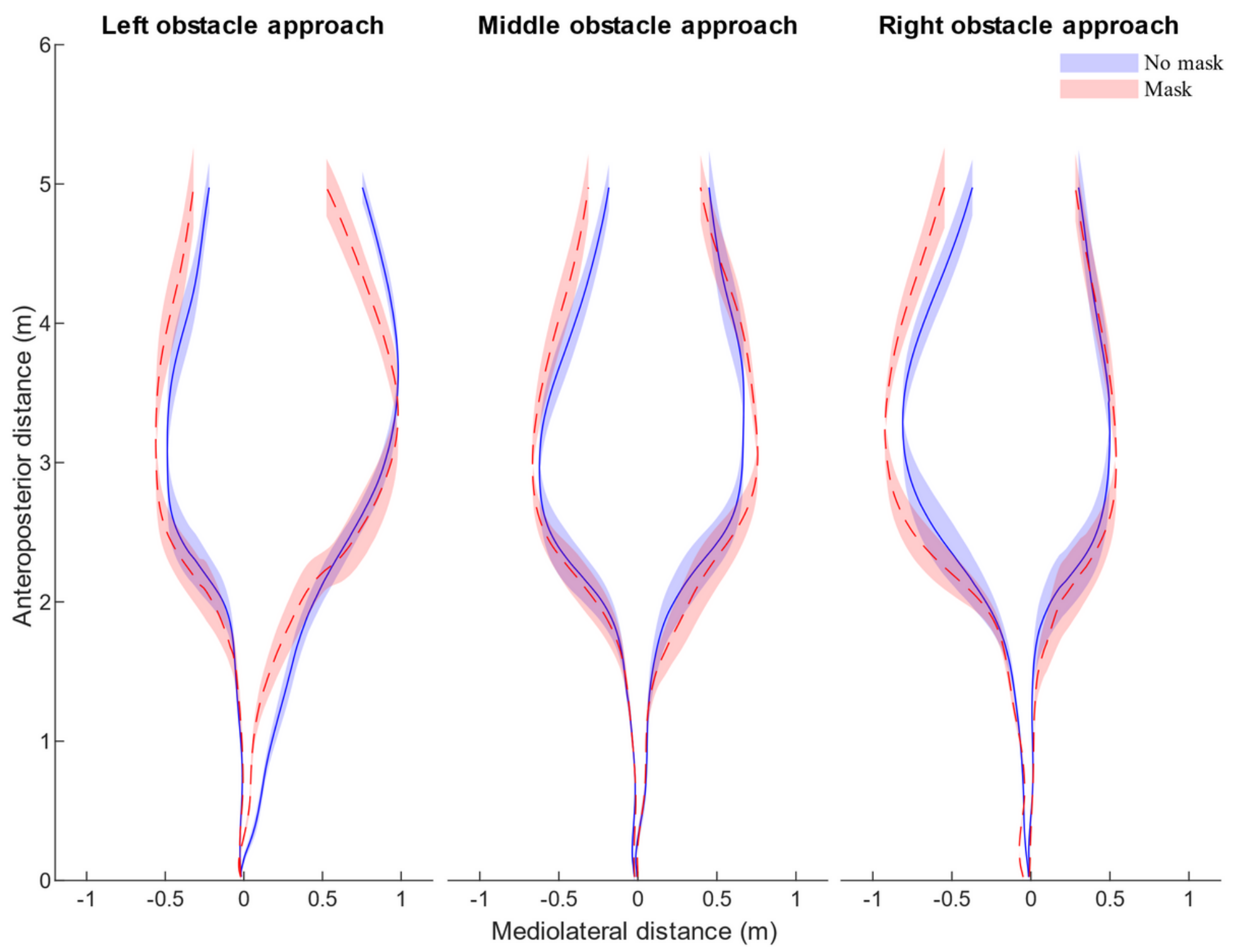

Figure 1

Trajectory traces (mean + 1SD) for the different obstacle approach conditions. Solid blue lines represent the no mask condition and dashed red lines represent the no mask condition. 

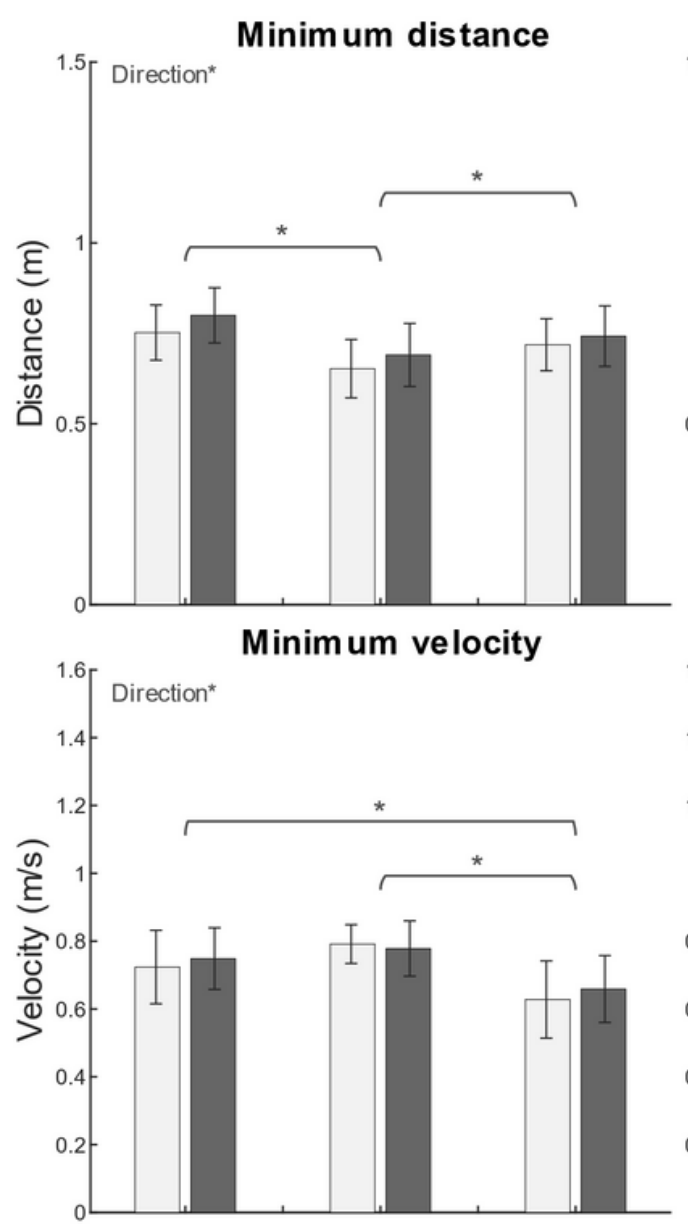

Fixation advancing VRP

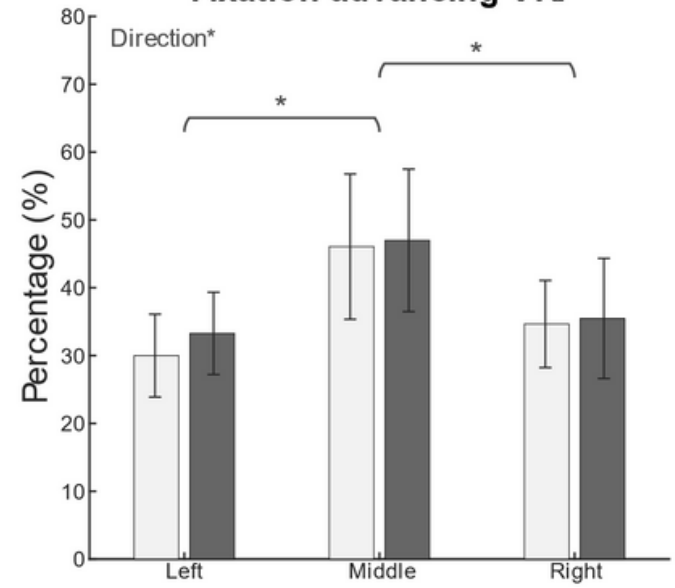

Maximum deviation

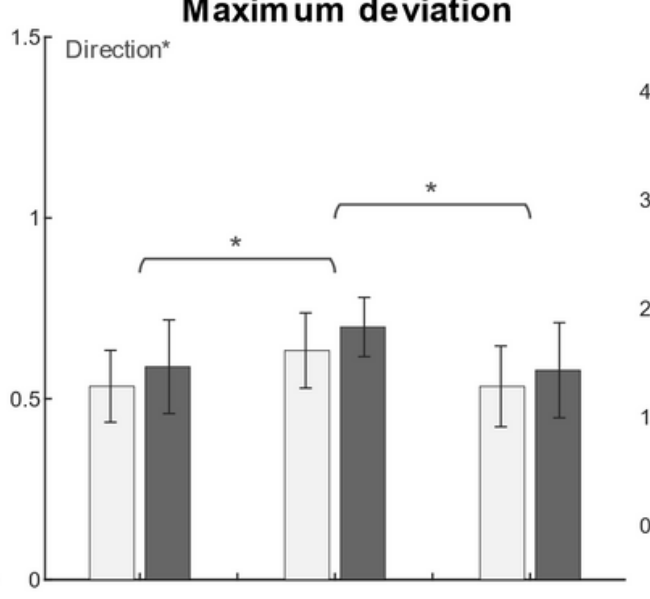

Average velocity

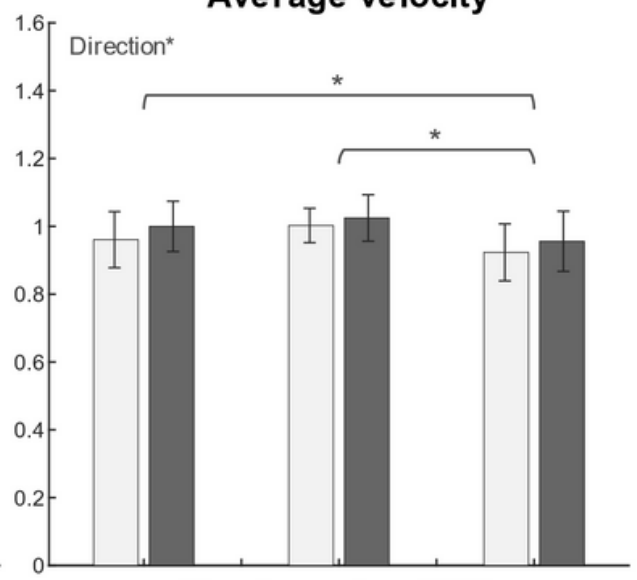

Fixation other VRPs

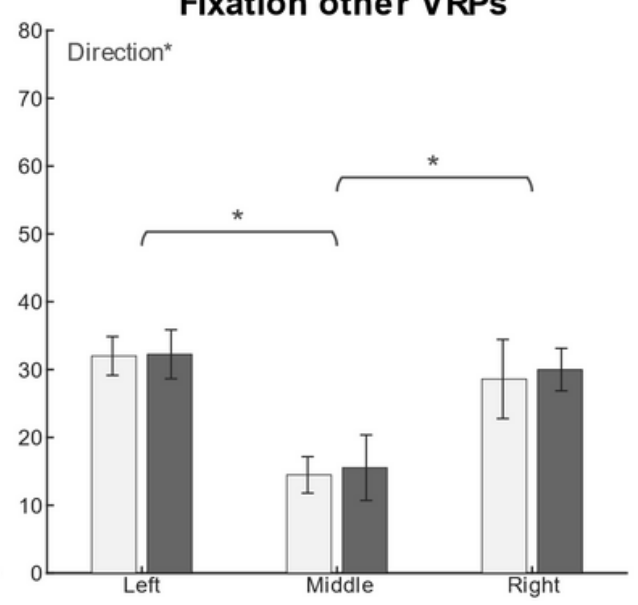

Onset distance

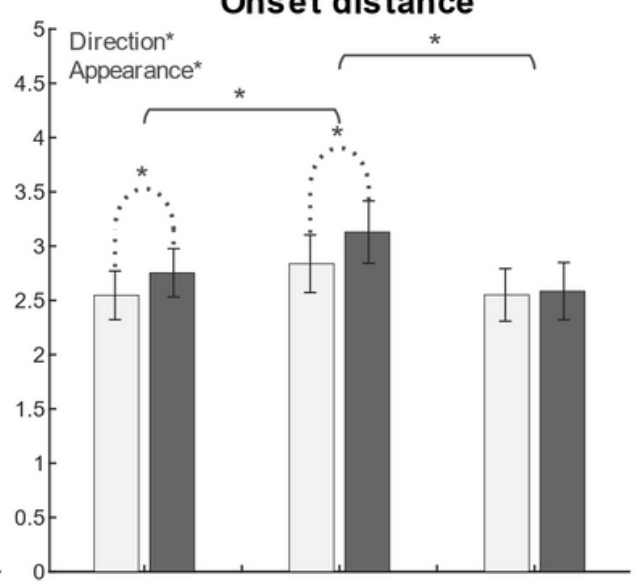

Maxim um velocity

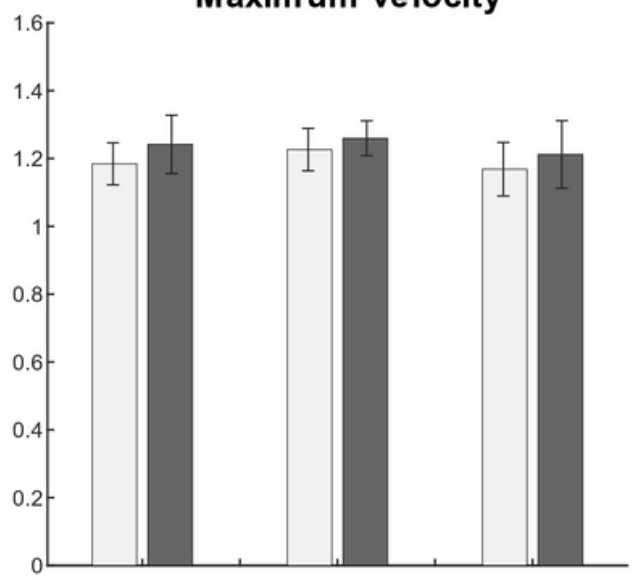

Fixation on VRP's head

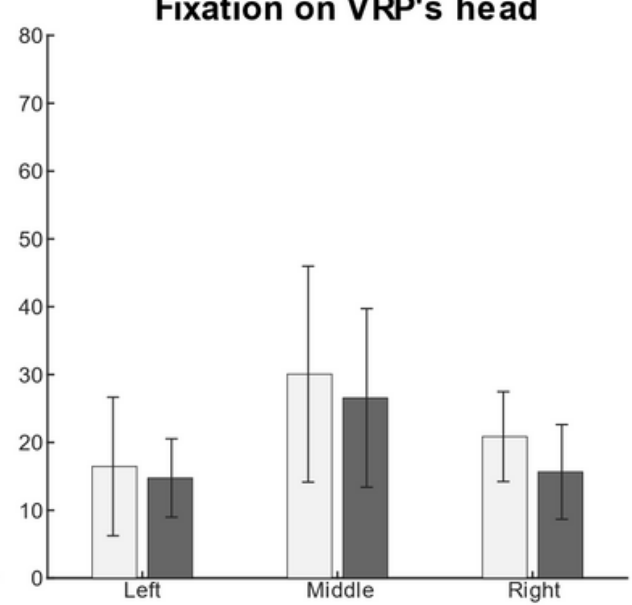

Figure 2

Mean \pm 1 SE for outcomes describing participants' behaviour when avoiding collisions with VRPs with a mask (light grey) and without (dark grey). The X-axis represents the direction of VRP approach. 


\section{Minimum distance}

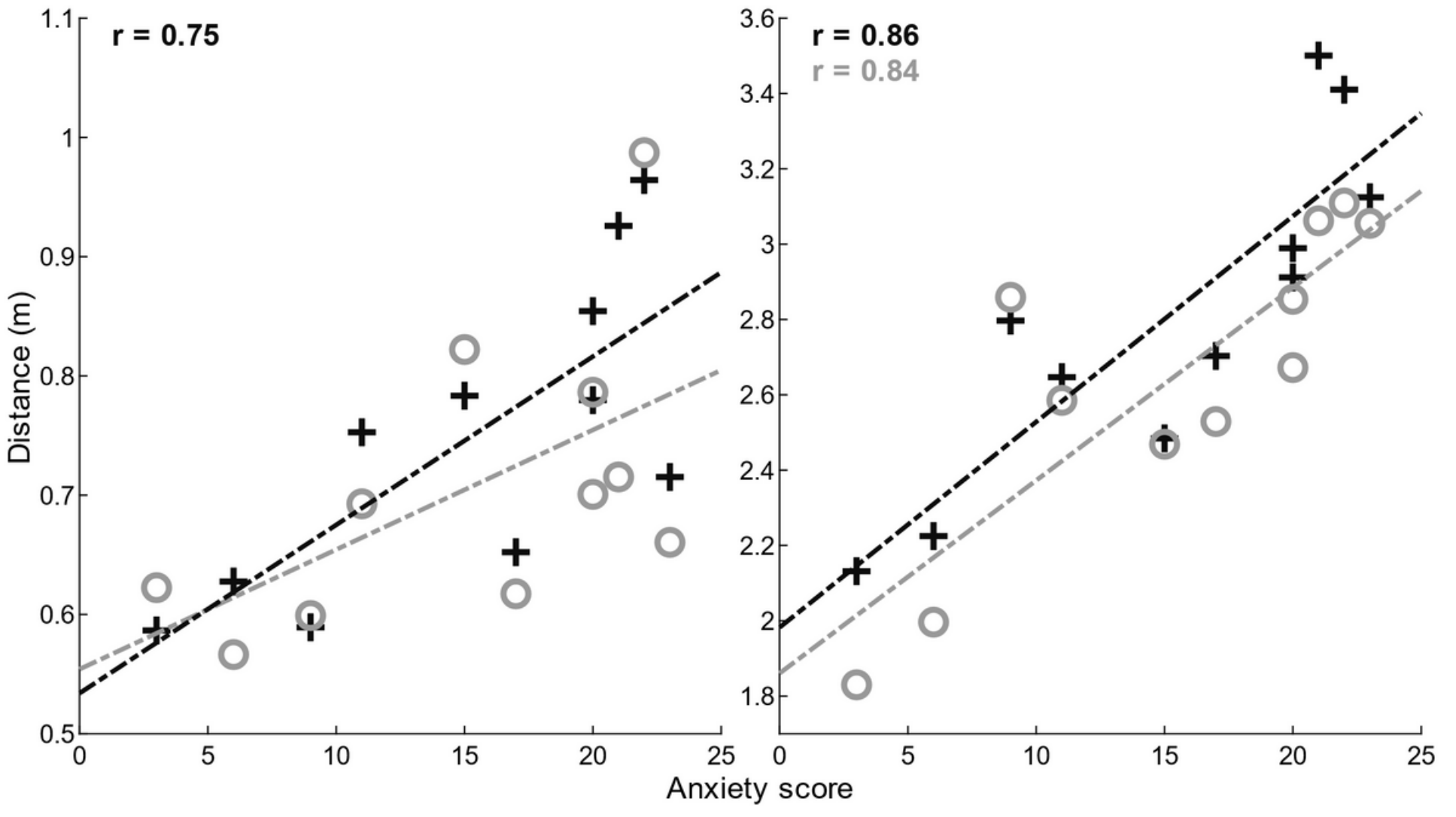

Figure 3

Scatter plots for correlations between anxiety score and measures of obstacle clearance (i.e., Minimum distance and Onset distance) and least squares regression lines for each condition. 


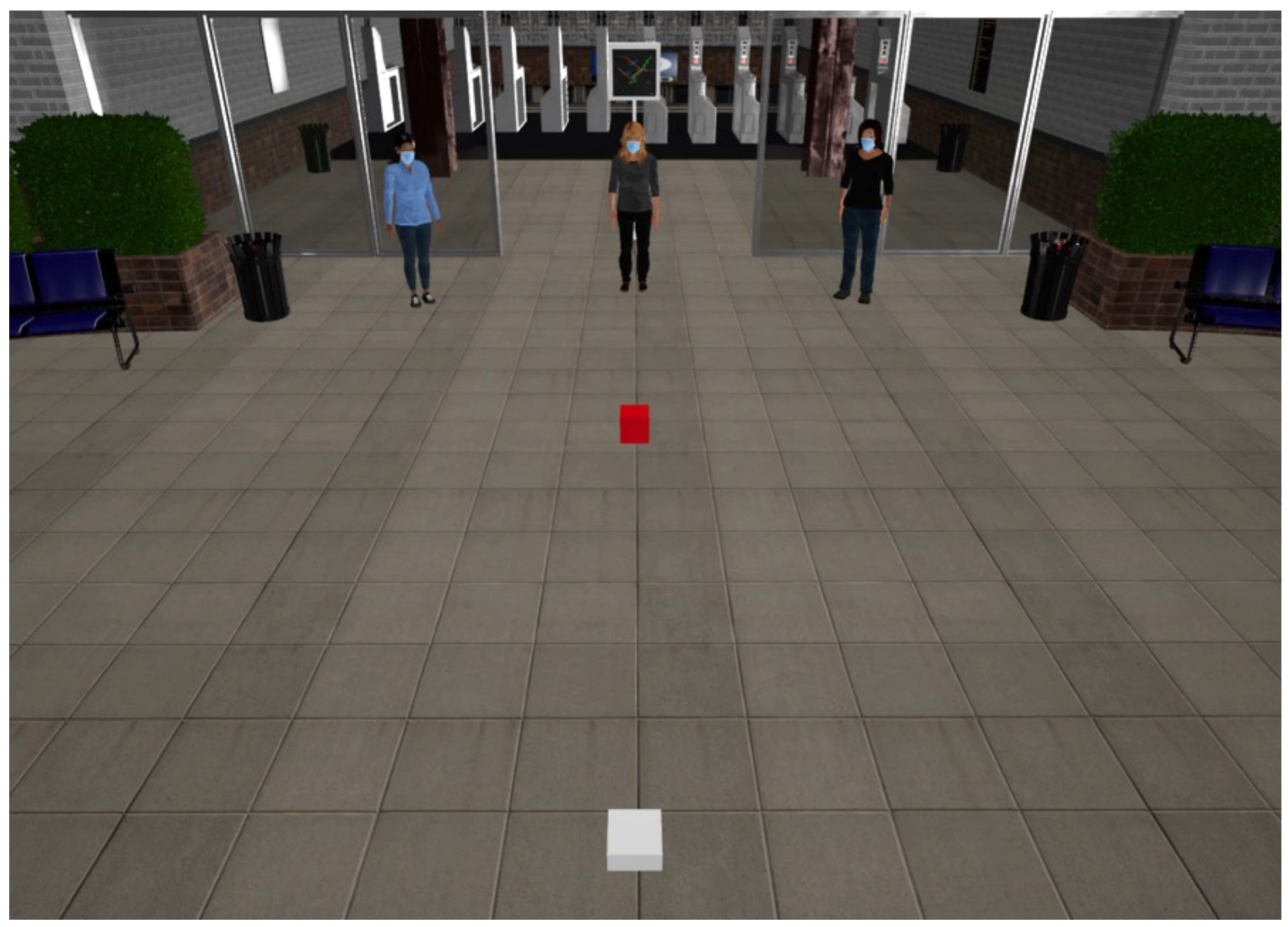

Figure 4

Perspective view of the virtual environment. At the start of each trial, participants stood at the initial position (white cube) facing three VRPs standing in front of a subway station entrance. The TPC (red cube), while invisible to the participant, illustrates the point at which a collision would occur if no walking adjustments were performed. 

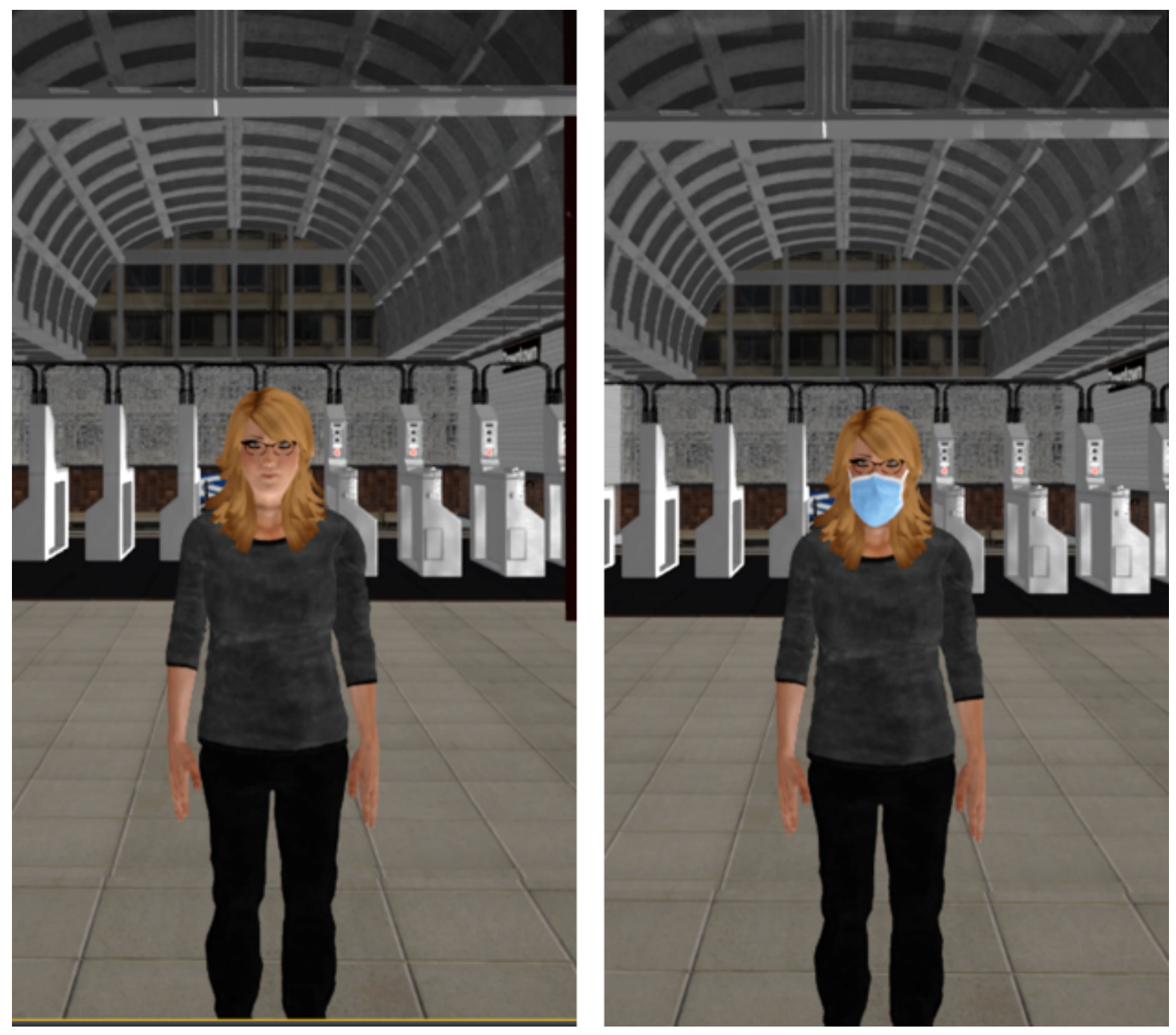

Figure 5

Example of a VRP without (left) and with a mask (right). 